M. J. TARLOW*

Department of Child Health, University of Aberdeen Medical School, Foresterhill, Aberdeen AB9 $2 Z D$.

*Correspondence to M.J.T., East Birmingham Teaching Unit, East Birmingham Hospital, Bordesley Green East, Birmingham B5 9ST.

\section{Erupted teeth in the newborn}

\section{6 members in a family}

A familial tendency to have erupted teeth in the newborn period was first described by Limrick in 1893 , and sporadic reports of up to 3 members in a family born with teeth have appeared since then. A family is described with 6 members having had erupted teeth in the newborn period probably following an autosomal dominant pattern of inheritance with limited penetrance.

\section{Case reports (Fig.)}

Case III.10. One of twins born after an uneventful pregnancy at 37 weeks' gestation. Presented at birth with an apparent eruption cyst. A lower central incisor erupted from this 3 days after birth and was removed without anaesthesia.

Case III.11. Twin sister of III.10 presented at birth with an identical lesion to her sister.

Case III.8. Sister of twins III.10 and 11 had a lower central incisor at birth. It was removed at 4 months because it was loose. The secondary dentition developed normally.

Case III.7. Two lower central incisors erupted when this otherwise normal girl was 1 month old.

Case III.1. An otherwise normal girl born with one lower central incisor. It was removed at 6 days because it was loose.

Case II.2. Normal eruption of teeth but with an imperforate anus and has a permanent colostomy.

Case II.3. Aunt of the affected members of the family. Born with 2 lower central incisors, an imperforate anus, and a rectovaginal fistula. Died at 5 months (in 1934) of septicaemia. No necropsy.

Case 1.2. Died at 51 of cerebral haemorrhage.

There is no history of natal or neonatal teeth in the father of cases III.1, 2, and 3, nor in the mothers of cases III.7 and cases III.8 to 11, nor in their families apart from their own children.

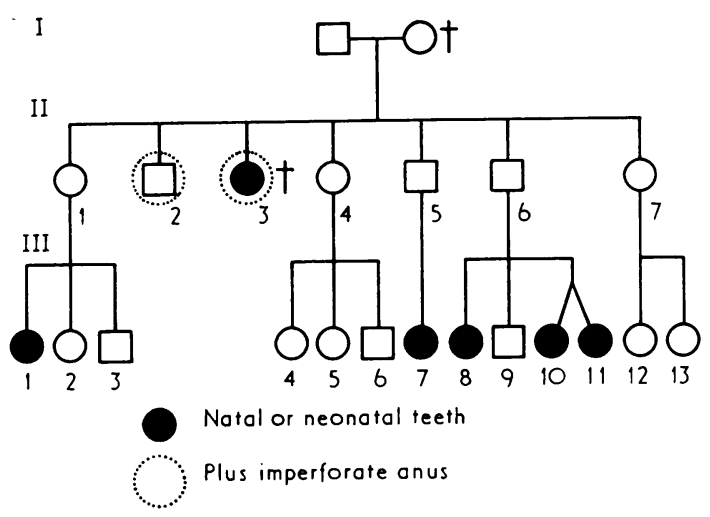

FIG.-Pedigree of family of whom 6 members had natally erupted teeth. $\quad$, death.

\section{Discussion}

Natal teeth have been known since ancient times. They were known to the Romans and to Shakespeare; Richard the Third was born with teeth. Some cultures regard them as a lucky event, particularly in Western Europe; however, in China they are regarded as an ill omen (Allwright, 1958). The incidence appears to be in the region of 1:2000 births in Great Britain (Gardiner, 1961).

Since the report of a mother, her son, and her sister's daughter by Limrick (1893), sporadic reports of up to 3 members of a family with natal teeth have appeared. 6 such reports were reviewed by Bodenhoff and Gorlin (1963). Gates (1946), largely on the basis of a family reported by Murray (1921), thought the condition was an 'irregular dominant'. Murray's family had associated hypertrophy of the nails and almost certainly had pachyonychia congenita. Gardiner (1961) found that 3 out of his 12 cases of natal teeth had a positive family history and Allwright (1958) found this in 2 of his series of 22.

No previous cases with a positive family history have been noted by paediatricians or dentists in Newcastle as far as we are aware, apart from 1 girl who had an erupted tooth at birth whose father, paternal uncle, and paternal aunt had teeth which erupted at 3 months of age.

Natal teeth are associated with 3 inherited syndromes: the Ellis-van Creveld syndrome is inherited in an autosomal recessive manner (polydactyly, short distal extremities, and nail hypoplasia), the Hallerman Streiff syndrome (microophthalmia, small beaked nose, and hypotrichia) is inherited as an autosomal dominant, and pachyonychia congenita (thickened nails and planto- 
palmar keratosis), is another autosomal dominant disorder (Smith, 1970). There is no evidence of any of these syndromes in the family reported here, whose inheritance seems to follow best an autosomal dominant pattern with incomplete penetrance. However, all the subjects affected in this family are girls, a fact that might favour a polygenic mode of inheritance. There is no sex difference in the condition as a whole (cases with and without a family history) in 2 large series (Bjuggren, 1973; Bodenhoff and Gorlin, 1963). No family trees have been published in previous papers on this subject, but boys seem to be affected in some families (Limrick, 1893; Bodenhoff and Gorlin, 1963; Bjuggren, 1973). The 2 members of generation II with an imperforate anus are of interest.

It is difficult to determine initially whether root formation will occur in natal or neonatal teeth. The majority will present problems only in breast feeding or by being excessively loose. If they remain loose there is a danger that they may exfoliate and be inhaled, and they are then best removed without anaesthesia. Bjuggren (1973) concludes that natal or neonatal teeth are rarely supernumerary to the deciduous dentition.

\section{Summary}

Erupted teeth in newborn infants is a well recognized, fairly common occurrence. Sporadic reports of several cases in a family have been described since 1893, and a proportion of natally erupted teeth seem to have a familial basis. A family with 6 members having erupted teeth at birth or shortly after is described, probably following an autosomal dominant pattern of inheritance with a limited penetrance.

We thank Dr. G. A. Neligan and Mr. G. H. Steel for their help in preparation of this paper.

REFERENCES

Allwright, W. C. (1958). Natal and neonatal teeth-a study among Chinese in Hong Kong. British Dental fournal, 105, 163.

Bjuggren, G. (1973). Premature eruption in the primary dentition-a clinical and radiological study. Svensk Tandläkare Tidskrift (Swedish Dental fournal), 66, 343.

Bodenhoff, J., and Gorlin, R. J. (1963). Natal and neonatal teeth. Pediatrics, 32, 1087.

Gardiner, J. H. (1961). Erupted teeth in the newborn. Proceedings of the Royal Society of Medicine, 54, 504.

Gates, R. R. (1946). Human Genetics, Vol. 1, p. 367. Macmillan, New York.

Limrick, O. E. B. (1893). Born with teeth. Lancet, 2, 965.

Murray, F. A. (1921). Congenital anomalies of the nails. British fournal of Dermatology, 33, 409.

Smith, D. W. (1970). Recognizable Patterns of Human Malformation, p. 284. Saunders, Philadelphia and London.

J. R. SiberT^ and J. R. Porteous

Princess Mary Maternity Hospital, Newcastle upon Tyne, and Department of Child Dental Health, University of Newcastle upon Tyne.

^Correspondence to Dr. J. R. Sibert, Department of Child Health, University Hospital of Wales, Heath Park, Cardiff CF4 4XW. 